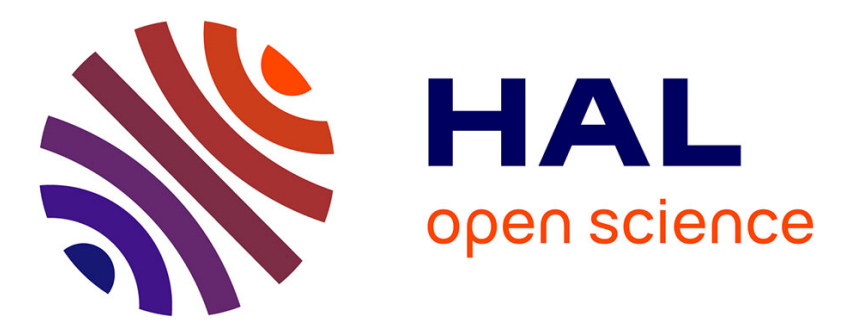

\title{
NEW IDENTIFICATION PROCEDURE FOR CONTINUOUS-TIME RADIO FREQUENCY POWER AMPLIFIER MODEL
}

Smail Bachir, Claude Duvanaud

\section{- To cite this version:}

Smail Bachir, Claude Duvanaud. NEW IDENTIFICATION PROCEDURE FOR CONTINUOUSTIME RADIO FREQUENCY POWER AMPLIFIER MODEL. Journal of Circuits, Systems, and Computers, 2010, 19 (06), pp.1259-1274. 10.1142/S0218126610006669 . hal-00781960

\author{
HAL Id: hal-00781960 \\ https://hal.science/hal-00781960
}

Submitted on 28 Jan 2013

HAL is a multi-disciplinary open access archive for the deposit and dissemination of scientific research documents, whether they are published or not. The documents may come from teaching and research institutions in France or abroad, or from public or private research centers.
L'archive ouverte pluridisciplinaire HAL, est destinée au dépôt et à la diffusion de documents scientifiques de niveau recherche, publiés ou non, émanant des établissements d'enseignement et de recherche français ou étrangers, des laboratoires publics ou privés. 
Journal of Circuits, Systems, and Computers

(C) World Scientific Publishing Company

\title{
New Identification Procedure for Continuous-Time Radio Frequency Power Amplifier Model
}

\author{
Smail Bachir* and Claude Duvanaud** \\ laboratoire d'Automatique et d'Informatique Industrielle de Poitiers LAII/ESIP \\ University of Poitiers \\ 40 avenue du recteur Pineau, 86022 Poitiers Cedex, France \\ *smail.bachir@univ-poitiers.fr, ** claude.duvanaud@univ-poitiers.fr \\ URL: http://laii.univ-poitiers.fr/
}

Received 07 September 2009

\begin{abstract}
In this paper, we present a new method for characterization of radio frequency Power Amplifier (PA) in the presence of nonlinear distortion which affect the modulated signal in the Radiocommunication transmission system. The proposed procedure uses a grey box model where PA dynamics are modelled with a MIMO continuous filter and the nonlinear characteristics are described as general polynomial functions, approximated by means of Taylor series. Using the baseband input and output data, model parameters are obtained by an iterative identification algorithm based on Output Error method. Initialization and excitation problems are resolved by an association of a new technique using initial values extraction with a multi-level binary sequence input exciting all PA dynamics. Finally, the proposed estimation method is tested and validated on experimental data.
\end{abstract}

Keywords: RF Power amplifier; parameter estimation; Nonlinear distortions; modeling; continuous time domain.

\section{Introduction}

There is increasing interest in high-level or behavioural modelling for simulation and design of complex radiocommunication sub-systems and components ${ }^{1,2}$. Radio frequency $(\mathrm{RF})$ nonlinear power amplifiers (PA), used to transmit the modulated signal, are classically characterized by two types of distortion: amplitude-to-amplitude $(\mathrm{AM} / \mathrm{AM})$ and amplitude-to-phase (AM/PM) characteristics. However, some uncertainty remains regarding the limitations and drawbacks of these commonly used approaches. These limitations are more evident in systems containing a significant degree of dynamical or when it is attempted to use this type of characterization with multiple carrier inputs and different modulation schemes.

The standard technique for PA modeling uses a nonlinear structure with fixed delay taps and complex coefficients ${ }^{3,4}$. A Volterra series ${ }^{1,5,6}$, Wiener and Hammerstein model ${ }^{2,7}$ are largely employed in quantifying Radio frequency (RF) effects. A number of algorithms exist performing the coefficient adaptations like the Least 
Mean Squares (LMS) technique which is the most commonly used algorithm ${ }^{8,9}$. However, the parameters computation for a nonlinear system is often difficult and time consuming for strongly nonlinear devices. The model considered in this paper is described on continuous-time domain. This structure is similar to PA discretetime representation which is including nonlinear transfer function and continuous filter ${ }^{3}$. The first branch is set to a memoryless amplitude (AM/AM) and phase (AM/PM) conversion. Conventionally, the power series model is used to considered these transfer functions. To describe a PA dynamics, an $n^{\text {th }}$ MIMO coupled filters is inserted. This element operates on modulating input and represents a low-pass equivalent in envelope signals ${ }^{10}$.

Model parameters are achieved using an iterative identification algorithm based on Output Error method.

During last two decades, there has been a new interest in Output Error techniques ${ }^{11,12,13,14}$. An overview of approaches is given in $^{8,15,16}$. Output Error (OE) methods are based on iterative minimization of an output error quadratic criterion by a Non Linear Programming (NLP) algorithm. This technique requires much more computation and do not converge to unique optimum. But, OE methods present very attractive features, because the simulation of the output model is based only on the knowledge of the input, so the parameter estimates are unbiased $^{9,17}$. Moreover, OE methods can be used to identify non linear systems. For these advantages, the $\mathrm{OE}$ methods are more appropriate in microwave systems characterization ${ }^{10}$. For PA identification, the parameters initialization and input excitation are very important to ensure global convergence. Then, we propose a new procedure for initialization search based on estimation of the nonlinear (AM/AM) and $(\mathrm{AM} / \mathrm{PM})$ functions decoupled from filter identification. A resulting value gives a good approximation of model parameters. Associated with a multi-level input excitation, this technique allows a fast convergence to the optimal values. Such an identification procedure for continuous-time domain in PA modeling does not seem to have been used previously.

The validation of this PA model is obtained for some digital modulation techniques in simulation and experimental investigations. Measured and estimated output signal are compared. Results show a good agreement and the possibility to PA characterization using continuous-time representation.

\section{PA Model description}

The nonlinear amplifier model used in this paper is an extension of the discrete timemodel at continuous representation ${ }^{3,18,19}$. The major disadvantage of the discrete representation is that the used parameters have no physical significance, contrary to continuous one where parameters keep their real aspect ${ }^{12,20}$. This is very important when advanced PA applications are considered such as linearization or real time control. 
The nonlinear block presented here operates on baseband quadrature I/Q timedomain waveforms. The complex low-pass equivalent (LPE) representation of the communication signal is used to avoid the high sampling rate required at the carrier frequency.

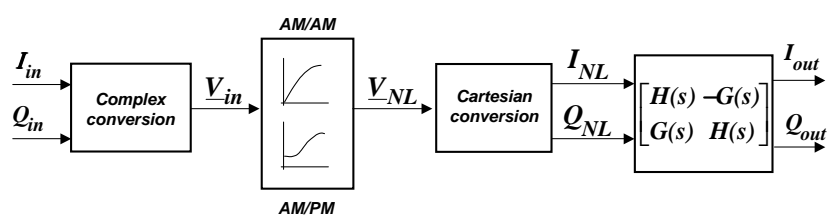

Fig. 1. Radio frequency power amplifier model

As shown in fig. 1, the two-box MIMO model includes a memoryless nonlinearity prior to an $n^{\text {th }}$ order Laplace filter. In this model, the first box is the AM/AM and $\mathrm{AM} / \mathrm{PM}$ conversions described PA nonlinearities. The second box is the frequency response which operates on the two baseband inputs $I / Q$.

\subsection{Nonlinear Static functions}

To take into account simultaneous gain and phase characteristics, amplifiers are traditionally modeled with a complex polynomial series ${ }^{2}$. Then, the complex envelope of the non linear output signal is approximated with the following baseband input/output relationship:

$$
\underline{V}_{N L}=\underline{V}_{i n} \cdot G\left(\left|\underline{V}_{i n}\right|^{2}\right)
$$

$\underline{V}_{i n}$ and $\underline{V}_{N L}$ are respectively the complex input and output voltage translated in baseband and expressed according the direct and quadrature $I / Q$ signals as:

$$
\left\{\begin{array}{l}
\underline{V}_{i n}=I_{i n}+j Q_{i n} \\
\underline{V}_{N L}=I_{N L}+j Q_{N L}
\end{array}\right.
$$

$G\left(\left|\underline{V}_{i n}\right|^{2}\right)$ is the complex gain of the amplifier, dependent of the magnitude of the input $\underline{V}_{i n}$. The complex gain is expressed with a polynomial function composed by even term which produce harmonic distortions inside the PA bandpass:

$$
G\left(\left|\underline{V}_{i n}\right|^{2}\right)=\sum_{k=0}^{P} \underline{c}_{2 k+1} \cdot\left|\underline{V}_{i n}\right|^{2 k}
$$

where $\underline{c}_{2 k+1}$ are the complex power series coefficients such as:

$$
\underline{c}_{2 k+1}=\alpha_{2 k+1}+j \beta_{2 k+1}
$$

The previous equations give the relationship between input and output baseband signals : 


$$
\left\{\begin{array}{l}
\underline{I}_{N L}=\sum_{k=0}^{P}\left(\alpha_{2 k+1} I_{i n}-\beta_{2 k+1} Q_{i n}\right) \cdot\left|\underline{V}_{i n}\right|^{2 k} \\
\underline{Q}_{N L}=\sum_{k=0}^{P}\left(\alpha_{2 k+1} Q_{i n}+\beta_{2 k+1} I_{i n}\right) \cdot\left|\underline{V}_{i n}\right|^{2 k}
\end{array}\right.
$$

The output quadrature signals depend on the both input quadrature terms and on the instantaneous input power.

\subsection{Continuous filter}

Such power amplifiers are more or less nonlinear which means that the output signal will be an amplified but distorted version of the input signal. Furthermore, these amplifiers have memory, i.e., the output signal does not only depend on the instantaneous value of the input signal. This results in a frequency-dependent transfer function and coupling effect between $I$ and $Q$ voices. These dynamical effect caused by the PA system behavior may be expressed with a MIMO differential model ${ }^{10}$. As shown in figure (1), the input to output relationships of this $n^{\text {th }}$ order filter can be written as:

$$
\left\{\begin{array}{l}
\frac{d^{n}}{d t^{n}} I_{\text {out }}+\sum_{k=0}^{n-1} a_{k} \frac{d^{k}}{d t^{k}} I_{\text {out }}=\sum_{k=0}^{m} b_{k} \frac{d^{k}}{d t^{k}} I_{N L}-\sum_{k=0}^{m^{\prime}} b_{k}^{\prime} \frac{d^{k}}{d t^{k}} Q_{N L} \\
\frac{d^{n}}{d t^{n}} Q_{\text {out }}+\sum_{k=0}^{n-1} a_{k} \frac{d^{k}}{d t^{k}} Q_{\text {out }}=\sum_{k=0}^{m^{\prime}} b_{k}^{\prime} \frac{d^{k}}{d t^{k}} I_{N L}+\sum_{k=0}^{m} b_{k} \frac{d^{k}}{d t^{k}} Q_{N L}
\end{array}\right.
$$

where $I_{\text {out }}(t)$ and $Q_{\text {out }}(t)$ are the filter outputs.

The coefficients $a_{k}, b_{k}$ and $b_{k}^{\prime}$ are real scalars that define the model. Note that the filter structure is the same on the diagonal axes $I Q$ and inverse on the other component, which gives a coupled MIMO plant. Thus, the input-output relation can be expressed in Laplace-domain with the two transfer-functions $H(s)$ and $G(s)$, as so:

$$
\left\{\begin{array}{l}
H(s)=\frac{\sum_{k=0}^{m} b_{k} \cdot s^{k}}{s^{n}+\sum_{k=0}^{n-1} a_{k} s^{k}} \\
G(s)=\frac{\sum_{k=0}^{m^{\prime} b_{k}^{\prime} \cdot s^{k}}}{s^{n}+\sum_{k=0}^{n-1} a_{k} s^{k}}
\end{array}\right.
$$

where $s$ denotes the differential operator $s=\frac{d}{d t}$.

\section{Parameter identification of the PA model}

Parameter identification is based on the definition of a model. For power amplifier, we consider the previous mathematical model (Eqs. 1-6) and we define the parameter vector:

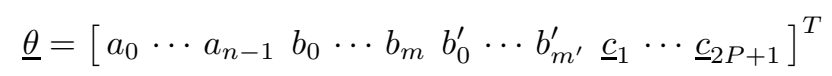

where [.] $]^{T}$ denotes transposition operation. 
A general principle of parameters estimation using Output Error technique is shown in Figure (2). Assume that we have measured $K$ values of input vector $\left(I_{\text {in }}(t), Q_{\text {in }}(t)\right)$ and output vector $\left(I_{\text {out }}^{*}(t), Q_{\text {out }}^{*}(t)\right)$ with $t=k \cdot T_{e}$ and $1 / T_{e}$ is the sampling rate. The identification problem is then to estimate the values of the parameters $\underline{\theta}$. Thus, we define the output prediction errors:

$$
\left\{\begin{array}{l}
\varepsilon_{I_{k}}=I_{\text {out }_{k}}^{*}-\hat{I}_{\text {out }_{k}}\left(\underline{\hat{\theta}}, I_{\text {in }}, Q_{\text {in }}\right) \\
\varepsilon_{Q_{k}}=Q_{\text {out }_{k}}^{*}-\hat{Q}_{\text {out }_{k}}\left(\underline{\hat{\theta}}, I_{\text {in }}, Q_{\text {in }}\right)
\end{array}\right.
$$

where predicted outputs $\hat{I}_{\text {out }_{k}}$ and $\hat{Q}_{\text {out }_{k}}$ are obtained by numerical simulations of the PA model and $\underline{\hat{\theta}}$ is an estimation of true parameter vector $\underline{\theta}$.

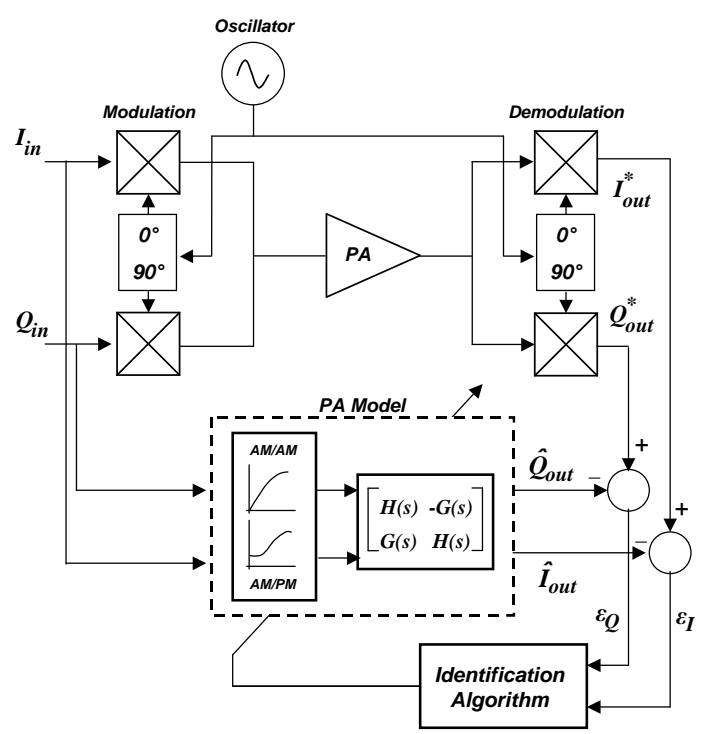

Fig. 2. PA identification scheme

As a general rule, parameter estimation with $\mathrm{OE}$ technique is based on minimization of a quadratic multivariable criterion defined as :

$$
J=\sum_{k=1}^{K}\left(\varepsilon_{I k}^{2}+\varepsilon_{Q k}^{2}\right)=\underline{\varepsilon}_{I}^{T} \underline{\varepsilon}_{I}+\underline{\varepsilon}_{Q}^{T} \underline{\varepsilon}_{Q}
$$

We obtain the optimal values of $\underline{\theta}$ by Non Linear Programming techniques. Practically, we use Marquardt's algorithm ${ }^{21}$ for off-line estimation:

$$
\underline{\hat{\theta}}_{i+1}=\underline{\hat{\theta}}_{i}-\left\{\left[J_{\theta \theta}^{\prime \prime}+\lambda \cdot I\right]^{-1} \cdot J_{\theta}^{\prime \prime}\right\}_{\hat{\hat{\theta}}=\underline{\theta}_{i}}
$$

$J_{\theta}^{\prime}$ and $J_{\theta \theta}^{\prime \prime}$ are respectively gradient and hessian such as:

$J_{\theta}^{\prime}=-2 \sum_{k=1}^{K}\left(\underline{\varepsilon}_{I_{k}}^{T} \cdot \underline{\sigma}_{I_{k, \underline{\theta}}}+\underline{\varepsilon}_{Q_{k}}^{T} \cdot \underline{\sigma}_{Q_{k, \underline{\theta}}}\right)$ 
$J_{\theta \theta}^{\prime \prime} \approx 2 \sum_{k=1}^{K}\left(\underline{\sigma}_{I_{k, \underline{\theta}}} \cdot \underline{\sigma}_{I_{k, \underline{\theta}}}^{T}+\underline{\sigma}_{Q_{k, \underline{\theta}}} \cdot \underline{\sigma}_{Q_{k, \underline{\theta}}^{T}}^{T}\right)$

$\lambda$ is the monitoring parameter,

The sensitivity $I Q$ functions $\underline{\sigma}_{I_{k, \underline{\theta}}}=\frac{\partial \hat{\hat{I}}_{\text {out }}}{\partial \underline{\underline{\underline{\theta}}}}$ and $\underline{\sigma}_{Q_{k, \underline{\theta}}}=\frac{\partial \hat{\hat{Q}}_{o u t}}{\partial \underline{\underline{\theta}}}$ are obtained, for each parameter, by partial differentiation ${ }^{23}$ of global PA model (Eqs. 1-6).

\subsection{Initialization problems}

An inherent problem of iterative search routines is that only convergence to a local minimum can be guaranteed. In order to converge to the global minimum, a good initialization is important. Usually, for engineering process, users have a good knowledge on physical parameters, necessary to initialize the iterative algorithm (Eq. 10). In our case, PA users have not sufficient information on parameter vector $\underline{\theta}$, especially on $\mathrm{AM} / \mathrm{AM}$ and $\mathrm{AM} / \mathrm{PM}$ parameters. It is then essential to define a global strategy which makes it possible to obtain approximative values of parameters. So we propose an optimal search method based on Equation Error techniques to achieve initial values of non linear and filter parameters.

\subsubsection{Non linear parameters initialization}

The first step consists in searching approximation of the complex parameters $\underline{c}_{2 k+1}$ using the envelope magnitude and phase distortions (Eqs. 1-3). Thus, the $\mathrm{AM} / \mathrm{AM}$ and $\mathrm{AM} / \mathrm{PM}$ characteristics are used to optimize a polynomial function by Least Mean Square (LMS) algorithm ${ }^{9}$. A solution for the coefficients is obtained by minimizing the mean-squared error between the measured $\left(I_{\text {out }}^{*}, Q_{\text {out }}^{*}\right)$ and the modeled output $\left(I_{\text {out }}, Q_{\text {out }}\right)$ under low frequency signal such as:

$$
\underline{\hat{\theta}}_{N L}=\left(\phi^{H} \phi\right)^{-1} \phi^{H} \underline{V}_{\text {out }}^{*}
$$

where :

$(.)^{H}$ denotes transpose-conjugate transformation

$\underline{\hat{\theta}}_{N L}=\left[\begin{array}{llll}\underline{c}_{1} & \underline{c}_{3} & \cdots & \underline{c}_{2 P+1}\end{array}\right]^{T}$ is the vector of polynomial parameters,

$\underline{V}_{\text {out }}^{*}$ is the measured output,

$\phi=\left[\begin{array}{llll}\underline{\varphi}_{1} & \underline{\varphi}_{2} & \cdots & \underline{\varphi}_{K}\end{array}\right]$ is the regression matrix,

$\underline{\varphi}_{k}=\left[\begin{array}{lll}V_{i n_{k}} & V_{i n_{k}}\left|V_{i n_{k}}\right|^{2} \cdots V_{i n_{k}}\left|V_{i n_{k}}\right|^{2 P}\end{array}\right]^{T}$ is the regression vector, and $\underline{V}_{i n_{k}}$ is a $k^{t h}$ sampled input.

Noted that for these estimations, the regression vector $\underline{\varphi}_{k}$ is not correlated with the measured output $\underline{V}_{\text {out }}^{*}$.

In practice, the $\mathrm{PA}$ characteristics is performed by a sinusoidal excitation applied on baseband inputs $I_{i n}$ and $Q_{i n}$ at fixed low frequency and high input level. In 
these conditions, the PA filtering effects are assumed negligible according to non linear dynamics. The input-output curves are obtained by measuring the output gain and phase as a function of input level.

\subsubsection{Filter parameters initialization}

The second step is the determination of initial values for the filter coefficients. They are obtained for an input signal with low input level and large frequency bandwidth. The signal distorsion is then negligible, which makes it possible to take into account only the linear filters $H(s)$ and $G(s)$ effects. Thus, we define the two filters parameters vectors:

$$
\begin{aligned}
\underline{\theta}_{f} & =\left[\begin{array}{llllllll}
a_{0} & a_{1} & \cdots & a_{n-1} & b_{0} & b_{1} & \cdots & b_{m}
\end{array}\right]^{T} \\
\underline{\theta}_{f}^{\prime} & =\left[\begin{array}{llllllll}
a_{0} & a_{1} & \cdots & a_{n-1} & b_{0}^{\prime} & b_{1}^{\prime} & \cdots & b_{m^{\prime}}^{\prime}
\end{array}\right]^{T}
\end{aligned}
$$

Parameter estimation is performed by iterative Instrumental Variable based on Reinitilized Partial Moments RPM method (see also ${ }^{10,13,23}$ ). Used for continuous filter identification, this technique is included in the integral methods class. The main idea of this class is to avoid the input-output time-derivatives calculation by performing integrations. In this class, the particularities of the $R P M$ method $^{\mathrm{a}}$ is the use of a time-shifting window for the integration and to perform an output noise filtering.

The main advantage of this estimation method to others is its relatively insensitivity to the initial conditions and rough system a priori knowledge.

\section{Simulation and experimental results}

\subsection{PA circuit and results}

The described power amplifier is a single stage structure composed of a MESFET device by Infineon (CLY 5). The nonlinear behaviour of the transistor is described by a table based model of the nonlinear drain current source associated to a junction model for the gate to source capacitance (Fig. 3).

The matching topology is designed to ensure optimum power and efficiency performance at the $900 \mathrm{MHz}$ operating frequency. The output matching has a low pass $\mathrm{T}$ structure composed of a capacitor to ground and two transmission lines.

Filter identification algorithm needs large frequency bandwidth excitation signal to provide appropriate estimation. Indeed, modulated signals are required to

${ }^{\text {a Contsid }}$ Matlab toolbox including the $R P M$ estimation method can be downloaded from http://www.cran.uhp-nancy.fr/contsid/. The ivrpm function allows to obtain model estimation by iterative Instrumental Variable. 


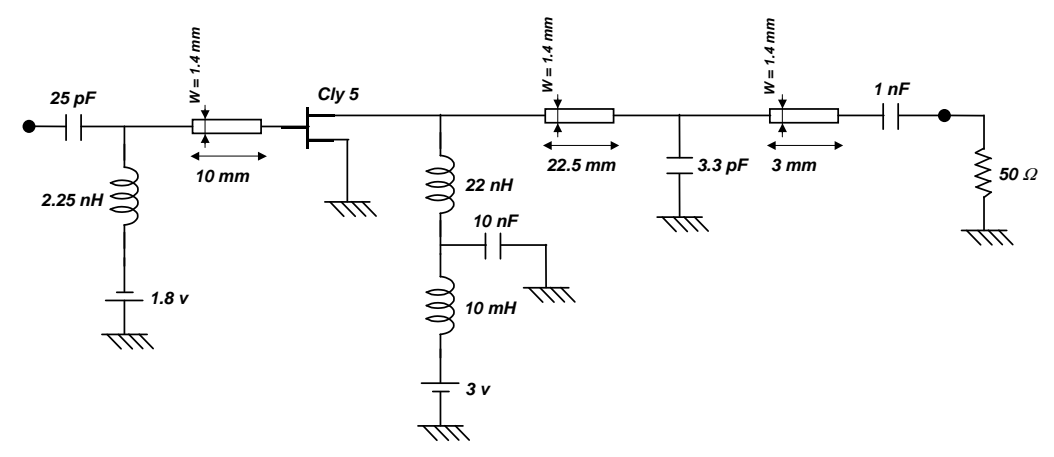

Fig. 3. PA circuit implemented on ADS software

excite both steady-state (low frequency) and process dynamics (medium to high frequency). This excitation is performed with a Pseudo Random Binary Sequence (P.R.B.S) baseband pulse as the input modulation to the transmitter. The identification procedure is performed in three steps : Initialization of nonlinear parameters, initialization of filter parameter and global identification of the PA's model.

Step 1 Nonlinear parameters $\underline{\theta}_{N L}$ are extracted from the input/output transfer function. The AM/AM and AM/PM measured characteristics are obtained by sweeping the power level of an input signal at a frequency located at the center of the PA bandwidth.
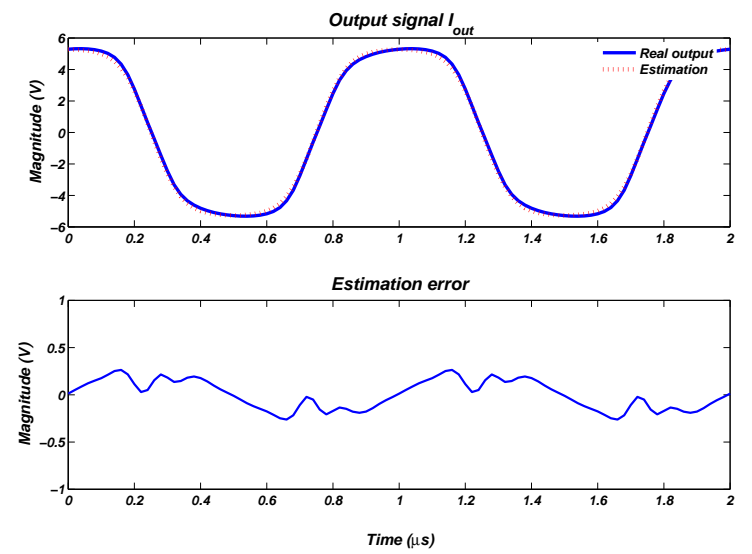

Fig. 4. (a) Comparison of time-domain measurement and estimation. (b) Estimation error

In our case, we used the $3^{\text {th }}$ order complex polynomial:

$$
\underline{V}_{N L}=\left(\underline{c}_{1}+\underline{c}_{3} \cdot\left|\underline{V}_{i n}\right|^{2}+\underline{c}_{5} \cdot\left|\underline{V}_{i n}\right|^{4}\right) \cdot \underline{V}_{i n}
$$


Thus, we define the estimated parameter complex vector:

$$
\underline{\theta}_{N L}=\left[\begin{array}{lll}
\underline{c}_{1} & \underline{c}_{3} & \underline{c}_{5}
\end{array}\right]^{T}
$$

After running a $L M S$ algorithm (Eq. 11), we obtained :

$$
\left\{\begin{array}{l}
\underline{\hat{c}}_{1}=6.9795-j 1.5681 \\
\underline{\hat{c}}_{3}=-2.5643+j 7.7424 .10^{-1} \\
\underline{\hat{c}}_{5}=4.9403 .10^{-1}-j 1.1504 .10^{-1}
\end{array}\right.
$$

Figure (4) allows a comparison between measured $I$ output waveforms and its estimation. As can be seen, even if the amplifier is driven near saturation, the $L M S$ algorithm converge to the optimum values with a maximum output estimation error less than $0.4 \mathrm{~V}$.

Step 2 The initial values $a_{i}, b_{i}$ and $b_{i}^{\prime}$ of the linear filters $H(s)$ and $G(s)$ are obtained by applying a Pseudo Random Binary Sequence (PRBS) signal with small amplitude level. To evaluate the efficiency of parameters estimator, we compared simulated output with real data of channel $I$ at the end of the identification procedure (Fig. 5). We can show that the estimation error (or residuals) is negligible and dont exceed $24 \mathrm{mV}$.
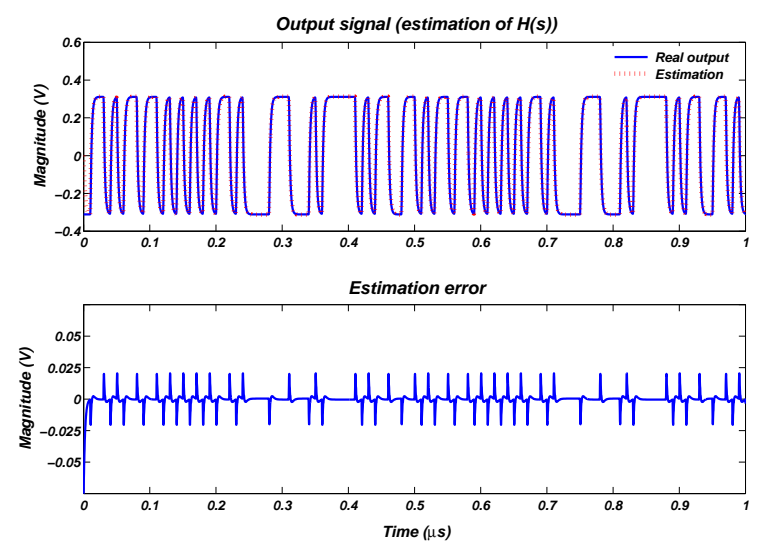

Fig. 5. Comparison between real and estimation data

The filter form is achieved using the RPM method for different plants. A quadratic error comparison allows to obtain an appropriate order. Then, for a $1^{\text {st }}$ order filters, the $R P M$ algorithm gives the parameters values:

$$
\begin{aligned}
\hat{H}(s) & =\frac{4.75 .10^{8}}{s+4.75 .10^{8}} \\
\hat{G}(s) & =\frac{2.51}{s+4.75 .10^{8}}
\end{aligned}
$$


The dynamic behavioral of the PA system can be described by a MIMO coupled filter. The real filter $H(s)$ and the obtained filter $\hat{H}(s)$ characteristics are represented in figure (6) by the gain and phase curves. The cut off frequency of the filter is around $76 \mathrm{MHz}$.
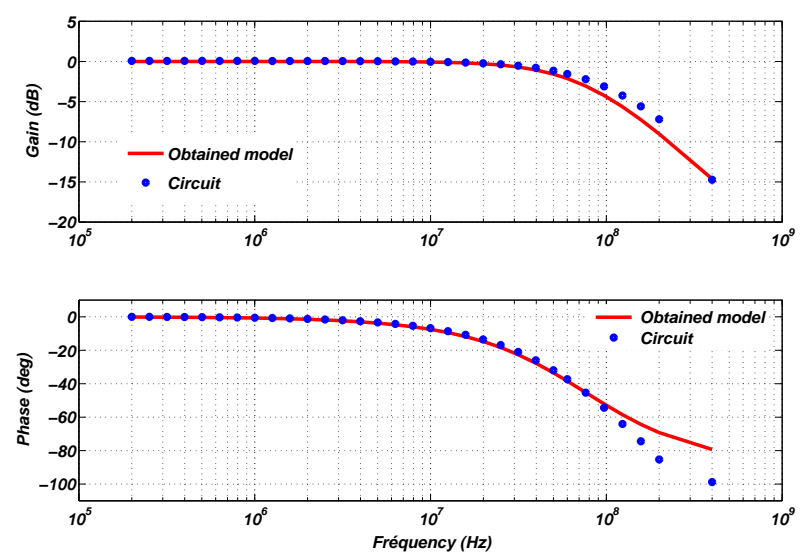

Fig. 6. Comparison between a frequency responses of the PA circuit and the obtained filter

Step 3 The model parameters obtained in the previous steps will be used to initialize the nonlinear identification algorithm.
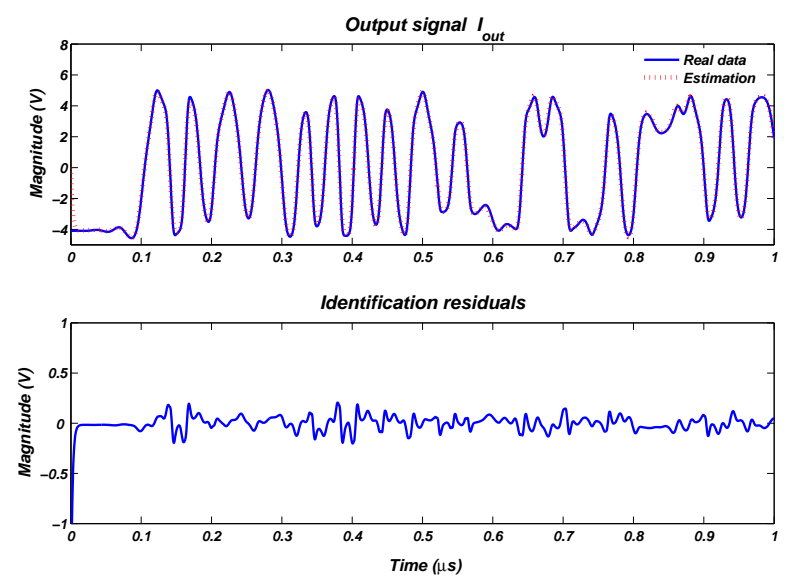

Fig. 7. Estimation of global PA model 
The unknown system in this case is the global PA model. The measurements are performed by an input signal obtained from the adding of some P.R.B.Sequences at different levels. The aim is to drive the amplifier in its overall level range (linear and non linear area). After 8 iterations, we obtain the following parameters:

$$
\underline{\hat{\theta}}=\left\{\begin{array}{l}
\hat{\underline{c}}_{1}=6.8745-j 1.5642 \\
\hat{\underline{c}}_{3}=-2.3634+j 7.8912 .10^{-1} \\
\hat{\underline{c}}_{5}=4.4974 .10^{-1}-j 1.1540 .10^{-1} \\
\hat{a}_{0}=4.7493 .10^{8} \\
\hat{b}_{0}=4.7493 .10^{8} \\
\hat{b}_{0}^{\prime}=2.44
\end{array}\right.
$$

Model simulation with the achieved parameters exhibit good approximation of measured data (Fig. 7).

\subsection{PA setup}

The measurement setup is shown in Fig. 8. The power amplifier is a commercial ZHL-42 from Mini CiRcuits manufacturer. Input and output data are obtained from YOKOGAWA Digital Oscilloscope with a sampling period equal to 10 ns.

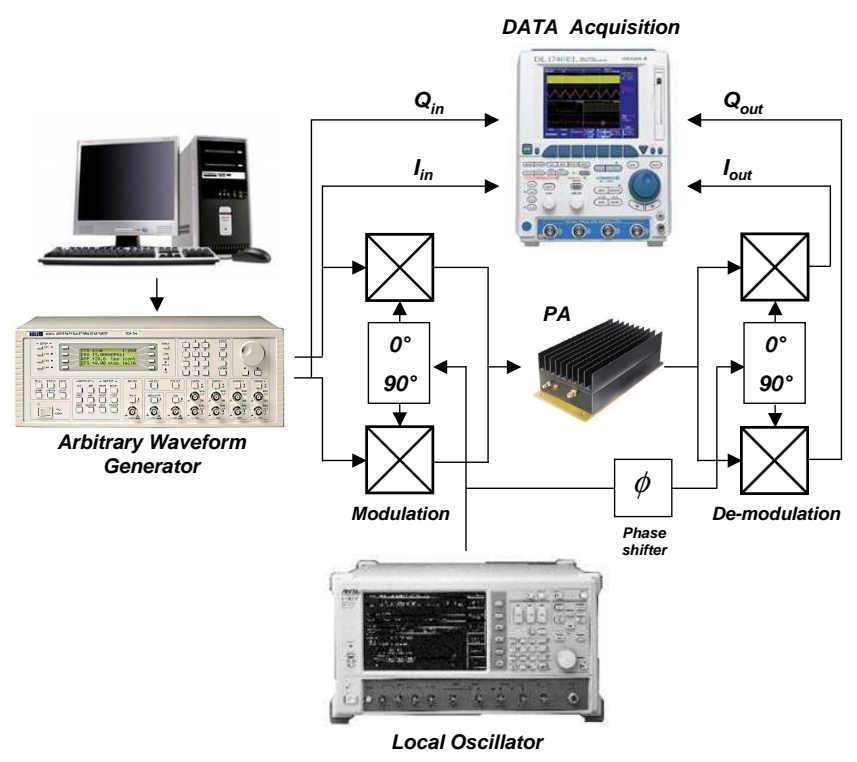

Fig. 8. PA setup

All data processing are carried using Matlab MathWorks then are down- 
loading to a Baseband Generator. The quadrature modulator AD8349 and demodulator AD8347 are inserted at the input and output of the PA. They are standard commercial units from Analog Devices.

Modulation signals $I$ and $Q$ are delivered by a TT $i 40 \mathrm{MHz}$ Arbitrary Waveform Generator connected to PC control. The local oscillator frequency is $900 \mathrm{MHz}$ obtained from Digital Modulation Signal Generator (ANRITsu MG 3660A).

$\mathrm{AM} / \mathrm{AM}$ and $\mathrm{AM} / \mathrm{PM}$ characteristics are given in figure (9). Thus, we can clearly see that the non linear behavioral of the amplifier is successfully described by a traditional third polynomial series.
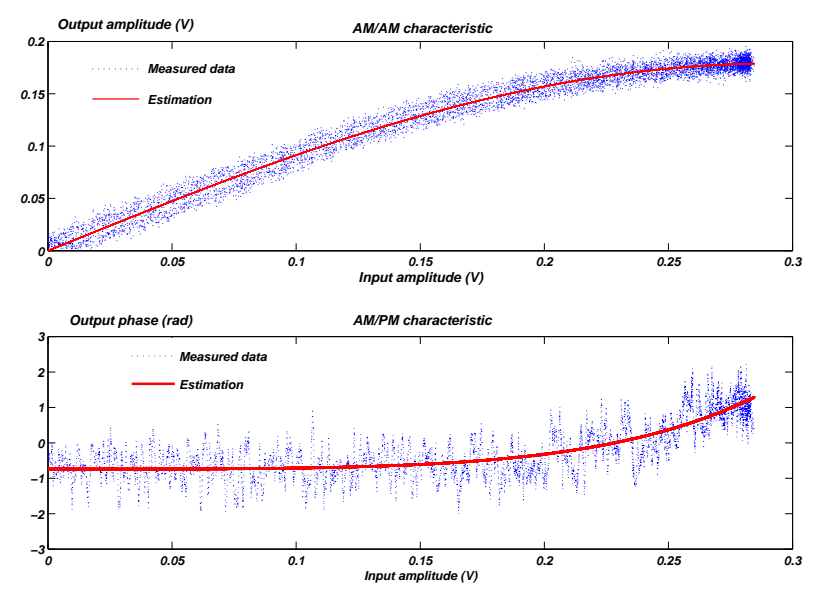

Fig. 9. Comparison between the measured and estimated AM/AM and AM/PM functions

For small power, figure (10) shows that the PA dynamic can be modeled as a $2^{\text {nd }}$ order resonant system. The RPM method gives the filters defined in the Laplace domain as:

$$
\left\{\begin{array}{l}
H(s)=\frac{b_{1} s+b_{0}}{s^{2}+a_{1} s+a_{0}} \\
G(s)=\frac{b_{0}^{\prime}}{s^{2}+a_{1} s+a_{0}}
\end{array}\right.
$$

where

$$
\begin{cases}\hat{a}_{0}=1.38 .10^{12} & \hat{a}_{1}=5.40 .10^{5} \\ \hat{b}_{0}=1.38 .10^{12} & \hat{b}_{1}=2.42 .10^{5} \\ \hat{b}^{\prime}{ }_{0}=12.53 & \end{cases}
$$

Figures (11.a) and (11.b) compare the simulated model output (dotted line) with the measured output for an excitation signal different of the one previously used for identification. As a test signal, we use a QPSK digitally modulated signal 
at rate of $5 \mathrm{Mbits} / \mathrm{s}$ and shaped with a raised cosine filter with a Rolloff factor of $\alpha=0.25$. It can be seen that the simulated output follows the measured one.
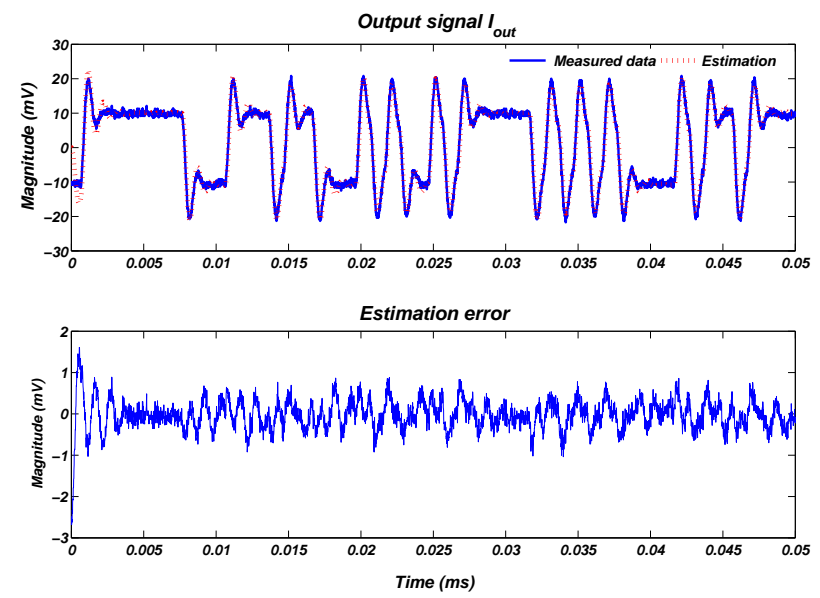

Fig. 10. (a) Input signal. (b) Comparison of time-domain measurement and estimation
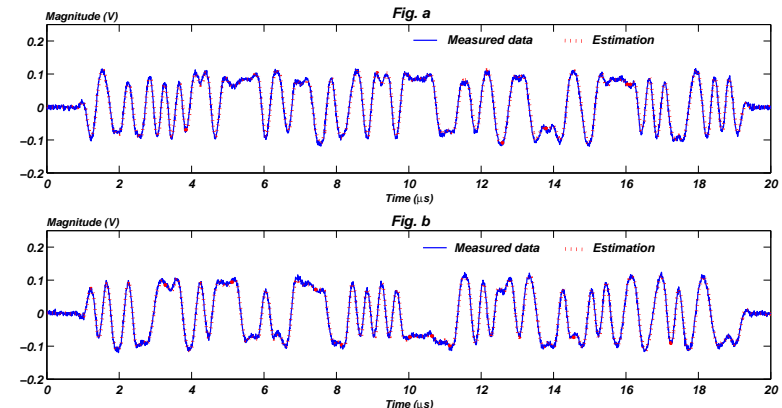

Fig. 11. Comparison of I/Q time-domain measurement and estimation for QPSK modulation

\section{Conclusion}

A model based on continuous-time representation is described which offers a simple way to modeling PA dynamics. This model is able of accounting the magnitude and phase amplifier nonlinearities such as the saturation effects.

Test results in simulation and experimentation illustrate the efficiency of this technique for use in off-line identification. The continuous approach was found to be 
accurate in predicting the dynamical response of the commercial amplifier. Estimation results show that the described amplifier acts like a resonant system coupled with a polynomial series.

The proposed technique is based on continuous time domain model. The model achieved can be used to develop a continuous baseband method for the compensation of nonlinearity of the RF front-end in a wireless transmitter.

\section{References}

1. S. C. Cripps, Advanced Techniques in RF power Amplifier Design, Artech House, Boston 2002.

2. P. B. Kennington, High-linearity RF Amplifier Design, Artech House, Boston 2000.

3. C. J. Clark, G. Chrisikos, M. S. Muha, A. A. Moulthrop and C. P. Silva, Time-domain envelope measurement technique with application to wideband power amplifier modeling, in IEEE Trans. Microwave Theory Tech. VOL. 46, NO. 12, pp. 2531-2540, Dec. 1998.

4. M. Isakson, D. Wisell and D. Ronnow, A Comparative Analysis of behavioral Models for RF Power Amplifiers, in IEEE Trans. Microwave Theory Tech., VOL. 54, No. 1, pp. 348-359, Junuary 2006.

5. A. Zhu, M. Wren, T. J. Brazil, An Efficient Volterra-based Behavioral Model for Wideband RF Power Amplifiers, MTT-S Int. Microwave Symposium Digest, VOL. 02, pp. 865- 868, 2003.

6. J. Xu, M. C. E. Yagoub, R. Ding and Q. J. Zhang, Neural-Based Dynamic Modeling of Nonlinear Microwave Circuits, in IEEE Trans. Microwave Theory Tech., VOL. 50, No. 12, pp. 2769-2780, December 2002.

7. T. Liu, S. Boumaiza, and F. M. Ghannouchi, Dynamic Behavioral modeling of $3 G$ Power Amplifiers Using real-Valued Time-Delay Neural Networks, in IEEE Trans. Microwave Theory Tech., VOL. 52, No. 3, pp. 1025-1033, March 2004.

8. P. Young, Parameter estimation for continuous-time models - a survey, Automatica, VOL. 17, No. 01, pp. 23-39, 1981.

9. L. Ljung, System identification: Theory for the user, $2^{\text {nd }}$ edition, Prentice Hall, USA, 1999.

10. M. Djamai, Contribution à la modélisation et à la linéarisation par prédistorsion numérique adaptative en bande de base des amplificateurs de radiocommunication, Ph.D. thesis, University of Poitiers, France 2008.

11. E. Walter and L. Pronzato, Identification of parametric models from experimental data, Communication and Control Engineering Series, Editions Springer, 1997.

12. H. Unbehauen and G.P. Rao, A review of identification in continuous-time systems, Annual Reviews in Control 22, pp. 145-171, 1998.

13. M. Mensler, H. Garnier and E. Huselstein, Experimental comparison of continuoustime model identification methods on thermal process, In: 12th IFAC Symposium on System Identification. Santa Barbara, California 2000.

14. H. Tulleken, Grey-box modeling and identification using physical knowledge and bayesian techniques, Automatica, VOL. 29, N0. 02, pp. 285-308, 1993.

15. I. D. Landau, Adaptive Control : The model reference aproach, Marcel Dekker. Control and Systems Theory. VOL. 08, 1979.

16. H. Unbehauen and G.P. Rao, Identification of continuous systems, Systems and control series. North-Holland, 1987.

17. J. Richalet, A. Rault and R. Pouliquen, Identification des processus par la mèthode du modèle, Gordon \& Breach, Thérie des systèmes, $\mathrm{N}^{o}$. 04, 1971. 
18. P. Stoica and T. Söderstrom, Instrumental-variable methods for identification of hammerstein systems, International Journal of Control, VOL. 35, NO. 3, pp. 459-476, 1982.

19. J. Vörös, Parameter identification of Wienner systems with discontinuous nonlinearities, Systems and Control Letters, VOL. 44, pp. 363-372, 2001.

20. V. Peterka, Trends and Progress in System Identification, Chapter Bayesian approach to system identification, pp. 239-304, Editions P. Eykhoff, Pergamon, Oxford, 1981.

21. D. W. Marquardt, An Algorithm for least-squares estimation of non-linear parameters, Soc. Indust. Appl. Math, VOL. 11, NO. 2, pp. 431-441, 1963.

22. M. Djamai, S. Bachir and C. Duvanaud, Behavioral modeling and digital predistortion of RF power amplifiers, Integrated Nonlinear Microwave and Millimetre-wave Circuits (INMMiC), Portugal, January 2006.

23. S. Bachir, S. Tnani, G. Champenois and J-C. Trigeassou, Control methods for Electrical machines, Chapter 8 : Diagnosis of induction machines by parameter estimation, pp. 245-268, ISTE Ltd and John Wiley \& Sons editions, London 2009.

24. H. F. Chen and L. Guo, Identification and Stochastic Adaptive Control, Birkhauser, Boston, 1991. 